\title{
GRAMICIDIN INDUCES THE FORMATION OF NON-BILAYER STRUCTURES IN PHOSPHATIDYLCHOLINE DISPERSIONS IN A FATTY ACID CHAIN LENGTH DEPENDENT WAY
}

\author{
C.J.A. VAN ECHTELD ${ }^{a}$, B. DE KRUIJFF ${ }^{b}$, A.J. VERKLEIJ ${ }^{b}, J^{\text {. LEUNISSEN-BIJVELT }}$ c and J. DE GIER $^{a}$ \\ "Department of Biochemistry. "Department of Molecular Biology and "Department of Molecular Cell Biologv. State University of \\ Utrechi, Transitorium III, Padualaan 8, NL-3584 CH Utrecht (The Netherlands)
}

Key words: Gramicidin; Hexagonal $H_{\|}$phase; Phosphatidylcholine; Fatty acid chain; Non-bilayer structure; Model membrane; $\left({ }^{\prime \prime} P-N M R\right)$

The hydrophobic peptide gramicidin is shown by ${ }^{31} \mathrm{P}-\mathrm{NMR}$, freeze-fracture electron microscopy and small-angle $\mathrm{X}$-ray diffraction, to induce a hexogonal $\mathrm{H}_{\mathrm{II}}$-phase lipid organization when incorporated in liquid crystalline saturated and unsaturated synthetic and natural phosphatidylcholines if the length of the fatty acids exceeds a 16 carbon atoms chain. The amount of hexagonally organized lipid increases with increasing fatty acid chain length. With phosphatidylcholines possessing shorter fatty acid chains, as well as with the longer phosphatidylcholines in the gel state, a lamellar organization results. Of the various possible models to explain the induction of the hexagonal $\mathrm{H}_{\mathrm{II}}$ phase by gramicidin, one in which gramicidin dimers span adjacent cylinders of the hexagonal $H_{\mid 1}$ phase seems most plausible. In phosphatidylcholines with intermediary chain lengths gramicidin induces intermediary structures, such as lipidic particles and possibly cubic phases. These experiments suggest that the balance between the length of the hydrophobic domain of a peptide and the membrane thickness is of critical importance for the structure of the membrane. In relation to this observation, the possible involvement of non-bilayer lipid structures in insertion and anchoring of membrane proteins is dicussed.

\section{Introduction}

Among the various factors $[1,2]$ that can modulate the macroscopic structure of membrane lipids, proteins and peptides form an intriguing class [3-9]. Due to its highly hydrophobic character, the helical dimer of the hydrophobic pentadecapeptide gramicidin may serve as a model for hydrophobic segments of membrane proteins [10]. It has been shown that in lamellar lipid structures gramicidin broadens the gel-to-liquid-crystalline transition region $[10,11]$, decreases chain order below the gel-to-liquid-crystalline transition temperature $\left(T_{c}\right)$ [11], and increases chain order above $T_{\mathrm{c}}$ $[11,12]$. At high gramicidin-to-lipid ratios (over $1: 4)$ only a decrease in chain order occurs, which is most obvious below $T_{c}[11,12]$. In general, gramicidin in phosphatidylcholine bilayers is known to preferably organize in $\mathrm{NH}_{2}$-terminal to $\mathrm{NH}_{2}$-terminal $\pi_{6}(L, D)$ helical dimers, stabilized by six hydrogen bonds [13-16].

When gramicidin is incorporated in dispersions of unsaturated phosphatidylethanolamines, it substantially decreases the bilayer to hexagonal $\mathrm{H}_{\mathrm{II}}$ phase transition temperature [17]. Apparently the gramicidin possesses a strong hexagonal $\mathrm{H}_{\mathrm{II}^{-}}$ phase-promoting ability. This observation is reinforced by the property of gramicidin to induce a hexagonal $\mathrm{H}_{11}$-phase organization even in dioleoylphosphatidylcholine [17], which is known to adopt exclusively a bilayer organization itself. In former studies on gramicidin with dimyristoylphosphatidylcholine and dipalmitoylphosphatidylcholine (1:10 molar ratio), only indications for 
bilayer organization have been observed $[10,18]$. This suggests that fatty acid chain lengths with corresponding hydrophobic volumes and mismatch with the length of the gramicidin dimer (25-30 A, Ref. 13) may be important parameters for the hexagonal $\mathrm{H}_{\mathrm{II}}$-phase-inducing property of gramicidin. We therefore have systematically studied the effect of gramicidin on phosphatidylcholines with different fatty acids.

\section{Materials and Methods}

Gramidicin from Bacillus brevis, which is a mixture of gramicidins A, B and C, was obtained from Sigma (St. Louis, MO, U.S.A.) and used without further purification.

1,2-Dilauroyl-sn-3-phosphocholine (12:0,12:0phosphatidylcholine), 1,2-dimyristoyl-sn-glycero3-phosphocholine (14:0,14:0-phosphatidylcholine), 1,2-dipalmitoyl-sn-glycero-3-phosphocholine (16:0,16:0-phosphatidylcholine), 1,2-distearoyl$s n$-glycero-3-phosphocholine $(18: 0,18: 0$-phosphatidylcholine), 1,2-dipalmitoleoyl-sn-glycero-3phosphocholine $\left(16: 1_{c}, 16: 1_{c}\right.$-phosphatidylcholine), 1,2-dioleoyl-sn-glycero-3-phosphocholine (18:1 $1_{c}, 18: 1_{c}$-phosphatidylcholine), 1,2-dielaidoyl$s n$-glycero-3-phosphocholine $\left(18: 1_{\text {tr }}, 18: 1_{\text {tr }}\right.$-phosphatidylcholine), 1,2-dierucoyl-sn-glycero-3-phosphocholine $\left(22: 1_{c}, 22: 1_{c}\right.$-phosphatidylcholine) were synthesized as described before [19]. Egg phosphatidylcholine was isolated from the total lipids of hen egg yolks, soya phosphatidylcholine was isolated from the total lipids of soya beans. All lipids were purified by HPLC [20] and were over $99 \%$ pure as determined by HPTLC. All other chemicals were of analytical grade.

Lipids were dissolved in chloroform and when desired, mixed with a chloroform/methanol $(1: 1$, $v / v)$ solution of gramicidin, evaporated to dryness under nitrogen and stored overnight under vacuum. Dispersions were prepared by adding $1.3 \mathrm{ml}$ of a $25 \%(\mathrm{v} / \mathrm{v}){ }^{2} \mathrm{H}_{2} \mathrm{O}$ buffer (100 mM NaCl$/ 25 \mathrm{mM}$ Tris/acetate/0.2 mM EDTA (pH 7.0)) to the dry film which contained approx. $60 \mu \mathrm{mol}$ phospholipid. The lipid films were allowed to swell for $3 \mathrm{~h}$ and subsequently gently vortexed above their gelto-liquid-crystalline transition temperature. This procedure appeared to be advantageous over a direct vigorous vortexing, because less small vesicles were formed as judged by freeze-fracture electron microscopy and ${ }^{31}$ P-NMR. When high gramicidin to lipid ratios (over 1:10) were applied, more vigorous vortexing was necessary to disperse the samples properly. For experiments on the influence of $\mathrm{K}^{+}$, gramicidin-lipid films (1:10 molar ratio) were dispersed in a $100 \mathrm{mM}$ Tris-acetate (pH 7.0) buffer, omitting the $\mathrm{NaCl}$ and EDTA. Aliquots of a $1 \mathrm{M} \mathrm{KSCN}$ solution were added during the NMR experiments up to a final concentration of $100 \mathrm{mM}$.

Proton noise decoupled ${ }^{31} \mathrm{P}-\mathrm{NMR}$ spectra were recorded at $81.0 \mathrm{MHz}$ on a Bruker WP 200 spectrometer as described before [17]. Accumulated free induction decays were exponentially multiplied, resulting in $50 \mathrm{~Hz}$ line broadening. $0 \mathrm{ppm}$ corresponds with the chemical shift position of sonicated egg phosphatidylcholine vesicles. Signal intensities of the various spectral components were obtained with an estimated accuracy of 3-5\% by computer subtraction methods using reference spectra consisting of only one spectral component. Freeze-fracturing was performed as outlined before [21]. Either samples, to which $25 \%(\mathrm{v} / \mathrm{v})$ glycerol was added to prevent freeze damage, were quenched conventionally in a mixture of solid and liquid nitrogen or they were quenched by jet freezing $[22,23]$ without the use of cryoprotectants. Small-angle X-ray diffraction was performed at room temperature using a Kratky camera with a $10 \times 0.2 \mathrm{~mm} \mathrm{Cu}-\mathrm{K} \alpha$ beam $(40 \mathrm{kV}, 23 \mathrm{~mA})$ and exposure times of $1-3 \mathrm{~h}$. The aqueous samples were mounted in slits $(12 \times 2 \times 2 \mathrm{~mm})$ inbetween two sheets of cellophane.

\section{Results}

In view of the presumption that fatty acid chain length may be an important parameter for the gramicidin-induced hexagonal $\mathrm{H}_{\mathrm{II}}$-phase organization of phosphatidylcholines, we have first tested this idea on phosphatidylcholines with long $22: 1_{c}$ fatty acid chains. Fig. 1A shows ${ }^{31} \mathrm{P}-\mathrm{NMR}$ spectra of an aqueous dispersion of 22:1 $, 22: 1_{c}$-phosphatidylcholine at various temperatures. The asymmetrical lineshape with a low-field shoulder and a high-field peak is characteristic for phospholipids in extended bilayers $[1,24]$. The residual chemical shift anisotropy $(\Delta \sigma)$, which was directly 


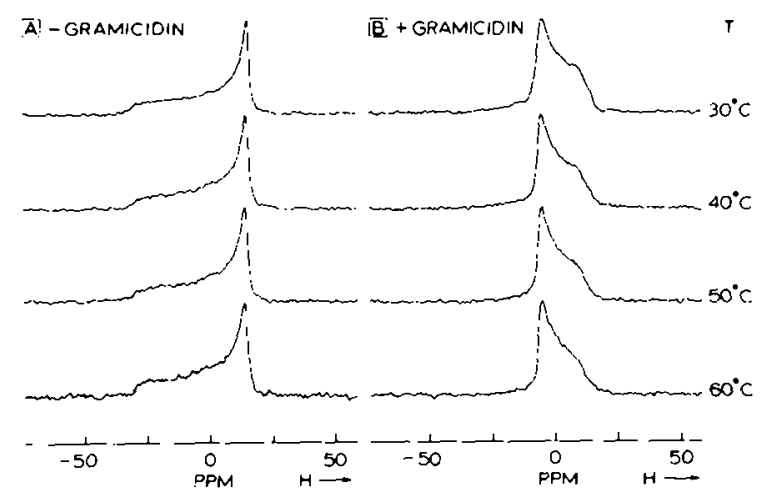

Fig. 1. Proton noise decoupled $81.0 \mathrm{MHz}{ }^{31}$ P-NMR spectra of aqueous dispersions of (A) $22: 1_{c} / 22: 1_{c}$-phosphatidylcholine and (B) a mixture of gramicidin:22: $1_{c} / 22: 1_{c}$-phosphatidylcholine $=1: 10$ at various temperatures indicated in the figure.

evaluated from the separation of the peak and shoulder resonance positions, varied slightly from $46 \mathrm{ppm}$ at $30^{\circ} \mathrm{C}$, to $42 \mathrm{ppm}$ at $60^{\circ} \mathrm{C}$ in accordance with the behaviour of other lamellar liquid crystalline phospholipids [24-26]. When gramicidin was incorporated in $22: 1_{c}, 22: 1_{c}$-phosphatidylcholine in a ratio of $1: 10$, the ${ }^{31}$ P-NMR spectral features changed drastically, as shown in Fig. 1B. The major part of the spectra is now determined by a low-field peak and a high-field shoulder separated by approx. $16 \mathrm{ppm}$ and appeared to be insensitive to temperature changes in the indicated region. The reversed asymmetry and the reduced $\Delta \sigma$ are characteristic for phospholipids organized in a hexagonal $H_{I I}$ phase, although $\Delta \sigma=16 \mathrm{ppm}$ is somewhat lower than the $\Delta \sigma=20 \mathrm{ppm}$ that is found for pure lipids that prefer a hexagonal organization, such as unsaturated phosphatidylethanolamines and $\mathrm{Ca}^{2+}$-cardiolipin $[27,28]$. The signal intensity on the low-field side of the peaks in the spectra of Fig. 1B indicates a minor amount of the phospholipid molecules in a lamellar organization. The high potency of gramicidin to induce the hexagonal $H_{11}$ phase when mixed with 22: $1_{c}, 22: 1_{c}$-phosphatidylcholine led us to choose this system to characterize further the hexagonal $\mathbf{H}_{\text {II }}$ phase of phosphatidylcholines.

Employing jet-freezing techniques we were able to visualize the hexogonal $\mathrm{H}_{11}$ phase of 22: $1_{c}, 22: 1_{c}$-phosphatidylcholine induced by gramicidin in a 10:1 ratio, whereas conventional freezing methods failed to reveal this phase. Fig. 2 shows the striated fracture pattern typical for the hexagonal $\mathrm{H}_{11}$ phase, as also was found before with a similar dispersion of $18: 1_{c}, 18: 1_{c}$-phosphatidylcholine: gramicidin $=10: 1$ [17]. The diameters of the hexagonal $\mathrm{H}_{\mathrm{II}}$ cylinders were found to be approx. $80 \AA$.

The occurrence of the hexagonal $\mathrm{H}_{\mathrm{II}}$ phase was also confirmed by small-angle $\mathrm{X}$-ray diffraction. Fig. 3 shows the X-ray diffraction patterns of $22: 1_{c}, 22: 1_{c}$-phosphatidylcholine with various amounts of gramicidin. The reflections are summarized in Table I. A dispersion of pure $22: 1_{c}, 22: 1_{c}$-phosphatidylcholine shows reflections with a ratio of $1 / 1: 1 / 2: 1 / 3$, typical for a lamellar organization (Fig. 3A). When gramicidin is incorporated in a 1:50 ratio, also a lamellar organization appears (Fig. 3B). However, when the ratio of gramicidin: $22: 1_{c}, 22: 1_{c}$-phosphatidylcholine is increased to $1: 10$ and $1: 5$ (Fig. 3C and D) reflections arise with ratio $1 / 1: 1 \sqrt{3}$. Although the higher-order reflections are very weak or absent, it clearly indicates a hexagonally organized lipid phase with a tube-to-tube distance of $75 \AA$. When the gramicidin-to-lipid ratio is increased further to $1: 2.5$, the diffraction bands become very diffuse, but a hexagonal organization seems to be maintained.

The gramicidin-concentration-dependent induction of the hexagonal $H_{I I}$ phase was further investigated using ${ }^{31} \mathrm{P}$-NMR. In Fig. $4{ }^{31} \mathrm{P}-\mathrm{NMR}$ spectra of dispersions of $22: 1_{c}, 22: 1_{c}$-phosphati-

\section{TABLE I}

SMALL-ANGLE X-RAY DIFFRACTION LINES OF $22: 1_{c} / 22: 1_{c}$-PHOSPHATIDYLCHOLINE-GRAMICIDIN MIXTURES

Reflection data are derived from densitometer tracings.

\begin{tabular}{llrrrr}
\hline & $\begin{array}{l}\text { Gramicidin: } \\
\text { lipid ratio }\end{array}$ & \multicolumn{1}{l}{ Reflections $(\dot{\mathrm{A}})$} & & \\
\hline A & 0 & 75 & - & 37.5 & $\sim 25$ \\
B & $1: 50$ & 78 & - & 39 & - \\
C & $1: 10$ & 74 & 43 & $-37 ?$ & - \\
D & $1: 5$ & 75 & 44 & - & - \\
E & $1: 2.5$ & -61 & -36 & - & - \\
Reflection ratio & $1 / 1$ & $1 / \sqrt{3}$ & $1 / 2$ & $1 / 3$ \\
\hline
\end{tabular}




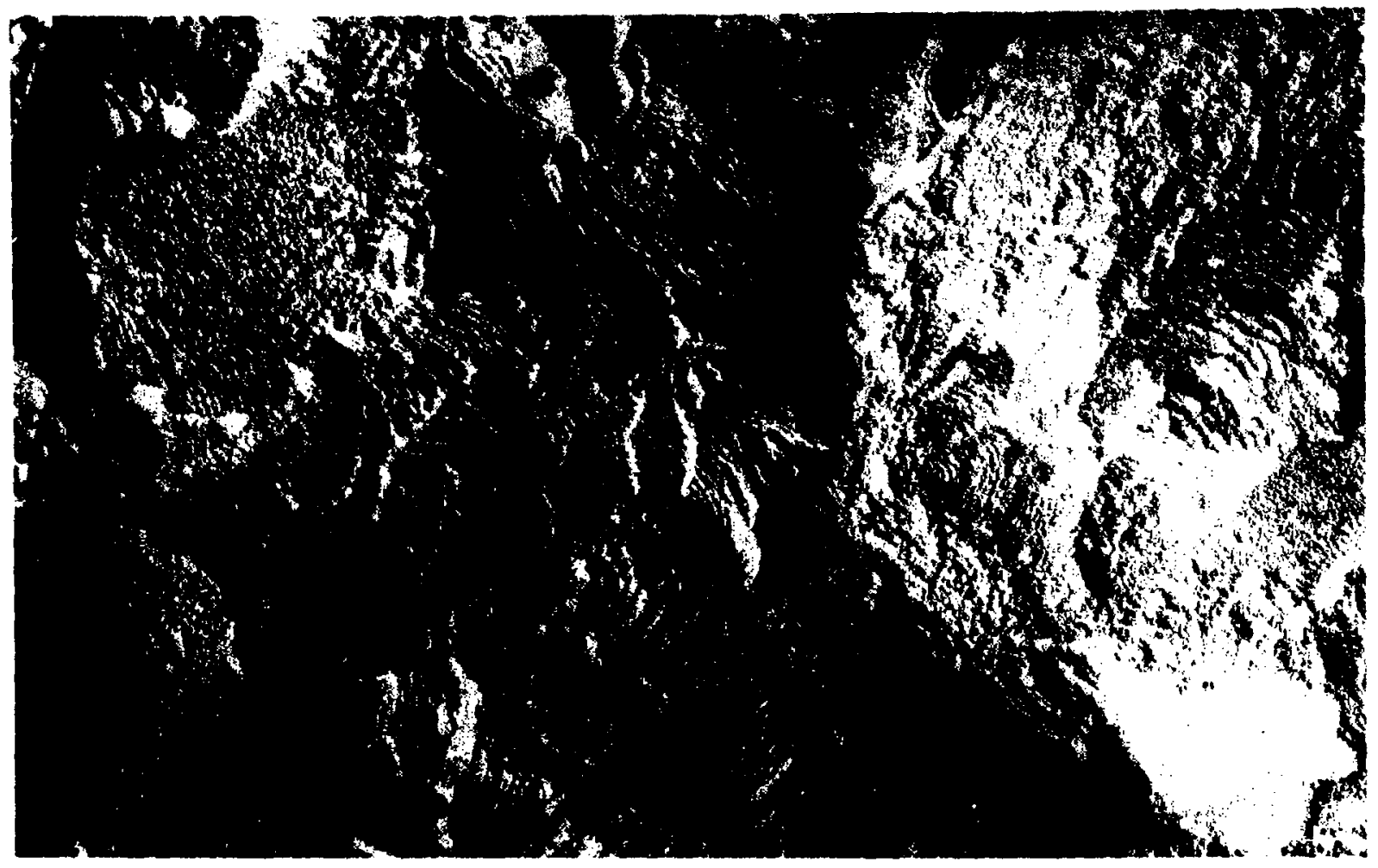

Fig. 2. Freeze-fracture electron micrograph of an aqueous dispersion of a mixture of gramicidin: $22: 1_{\mathrm{c}} / 22: 1_{\mathrm{c}}$-phosphatidylcholine $=$ $1: 10$. The sample was quenched from $30^{\circ} \mathrm{C}$ employing jet-freeze techniques. Magnification approx. $100000 \times$.

dylcholine with various amounts of gramicidin incorporated are presented. Incorporation of gramicidin causes a decrease in $\Delta \sigma$ of the bilayer component of the spectra from $47 \mathrm{ppm}$ to approx. $38 \mathrm{ppm}$ and an increase in linewidth, in fair agree-

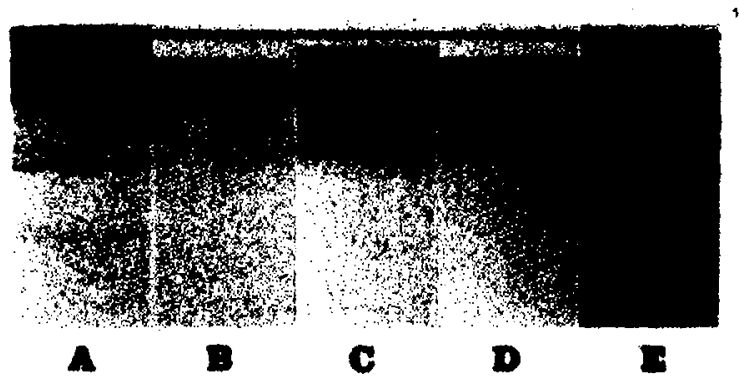

Fig. 3. Small-angle $\mathrm{X}$-ray diffraction patterns of aqueous dispersions of $22: l_{c} / 22: l_{c}$-phosphatidylcholine (A) and gramicidin: 22: $1_{c} / 22: 1_{c}$-phosphatidylcholine mixtures with molar ratios of, respectively, $1: 50(\mathrm{~B}) ; 1: 10(\mathrm{C}) ; 1: 5(\mathrm{D})$ and $\mathrm{I}: 2.5(\mathrm{E})$. ment with previous results [18]. This could be due to a conformational or motional change in the polar head group of the lipid molecules but can equally well be explained by a smaller average size of the liposomal structures [29]. When considering the fact that gramicidin indeed tends to reduce the size of the liposomal structures, as evidenced by freeze-fracturing [17], the latter explanation seems more appropriate.

A second, more striking feature of these spectra is the presence of a component indicating hexagonally $\mathrm{H}_{\mathrm{II}}$ organized lipid which is already noticeable at the $1: 50$ ratio. The amount of $22: 1_{c}, 22: 1_{c^{-}}$ phosphatidylcholine organized in the hexagonal $\mathrm{H}_{11}$ phase increases with increasing gramicidin content and is clearly concentration-dependent. However, samples with a gramicidin-to-lipid ratio of $1: 2.5$ behave anomalously in that the ${ }^{31} \mathrm{P}-\mathrm{NMR}$ spectra fail to indicate a substantial amount of hexagonally organized lipid. Instead, a less well defined, more symmetrical lineshape arises. 


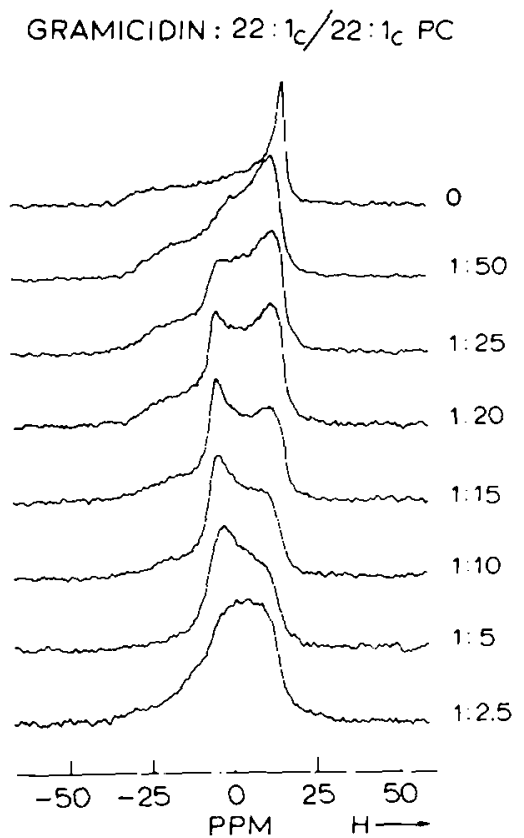

Fig. 4. Proton noise decoupled $81.0 \mathrm{MHz}{ }^{3 /} \mathrm{P}$ NMR spectra of aqueous dispersions of $22: 1_{c} / 22: 1_{c}$-phosphatidylcholine with increasing amounts of incorporated gramicidin at $25^{\circ} \mathrm{C}$. Molar ratios are indicated in the figure.

Freeze-fracturing of this latter sample shows areas of hexagonal $\mathrm{H}_{\mathrm{II}}$ phase, next to vesicles $(500-5000$ $\AA$ ) and small densely packed ill-defined structures (data not shown).

A quantitative interpretation of the ${ }^{31}$ P.NMR data is presented in Fig. 5. The amount of 22: $1_{c}, 22: 1_{c}$-phosphatidylcholine organized in the hexagonal $H_{11}$ phase reaches an apparent maximum of approx. $80 \%$ at a molar ratio of gramicidin: lipid $=1: 5$. Since we already have demonstrated that gramicidin induces the hexagonal $\mathrm{H}_{11}$ phases in 18: $1_{c}, 18: 1_{c}$-phosphatidylcholine [17] the gramicidin-concentration dependency of the induction of the hexagonal phase was also investigated for this lipid (see Fig. 5). Qualitatively, these samples exhibit the same behaviour, but quantitatively the effects of gramicidin are less pronounced and the maximum is reached at lower gramicidin contents for the $18: 1_{c}, 18: 1_{c}$-phosphatidylcholine. The quantitative differences of these results suppose a fatty acid chain length dependency on the extent of the induction of the hexagonal $\mathrm{H}_{1 \mathrm{I}}$ phase in diacylphosphatidylcholines by gramicidin.

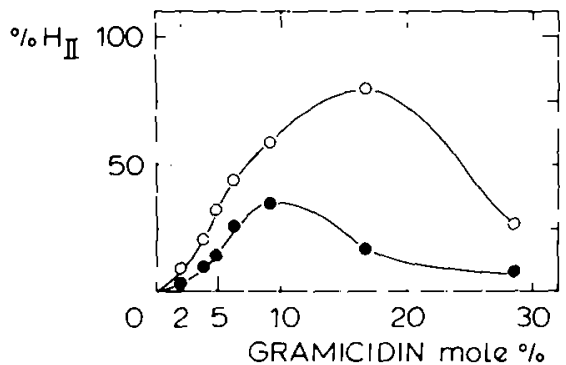

Fig. 5. Amount of phospholipid organized in a hexagonal $\mathrm{H}_{\mathrm{II}}$ phase in aqueous dispersions of mixtures of $(\mathrm{O}-\mathrm{O})$ $22: 1_{c} / 22: 1_{c}$-phosphatidylcholine and 18:1 $/ 18: 1_{c}$-phosphatidylcholine with various amounts of gramicidin as derived from ${ }^{3 \mathrm{I}} \mathrm{P}-\mathrm{NMR}$ spectra recorded at $25^{\circ} \mathrm{C}$ (see Fig. 4). Data points represent the mean of three different samples with a standard error ranging from 2-5\%.

Therefore, comparisons were made with other unsaturated diacylphosphatidylcholines.

Fig. 6 shows the ${ }^{31}$ P-NMR spectra of dispersions of various unsaturated diacylphosphatidylcholines in which gramicidin was incorporated in a 1:10 ratio. As can be seen, the onset of the induction of the hexagonal $\mathrm{H}_{\mathrm{II}}$ phase, which is most easily recognized by the resonance intensity at approx. $-6 \mathrm{ppm}$, coincides with a fatty acid chain length of 18 carbon atoms, although gramicidin is much more effective in inducing this

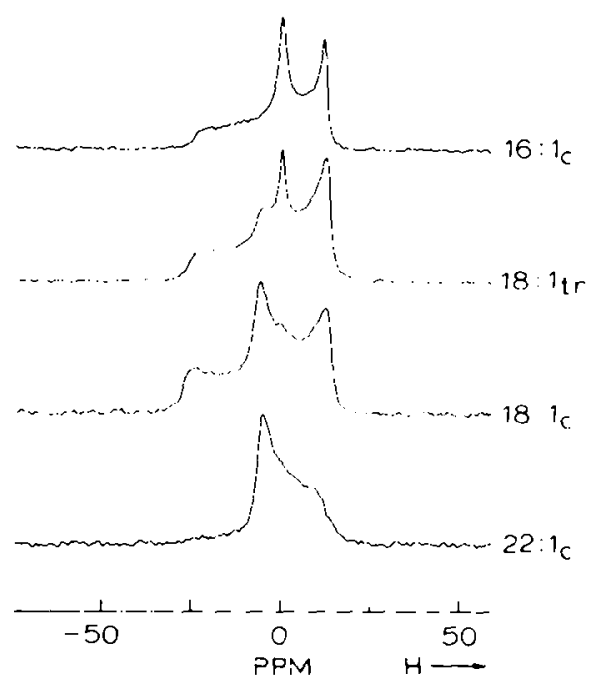

Fig. 6. Proton noise decoupled $81.0 \mathrm{MHz}{ }^{31} \mathrm{P}-\mathrm{NMR}$ spectra of aqueous dispersions of mixtures of gramicidin with various unsaturated diacylphosphatidylcholines in a $1: 10$ molar ratio at $25^{\circ} \mathrm{C}$. Fatty acid composition as indicated in the figure. 


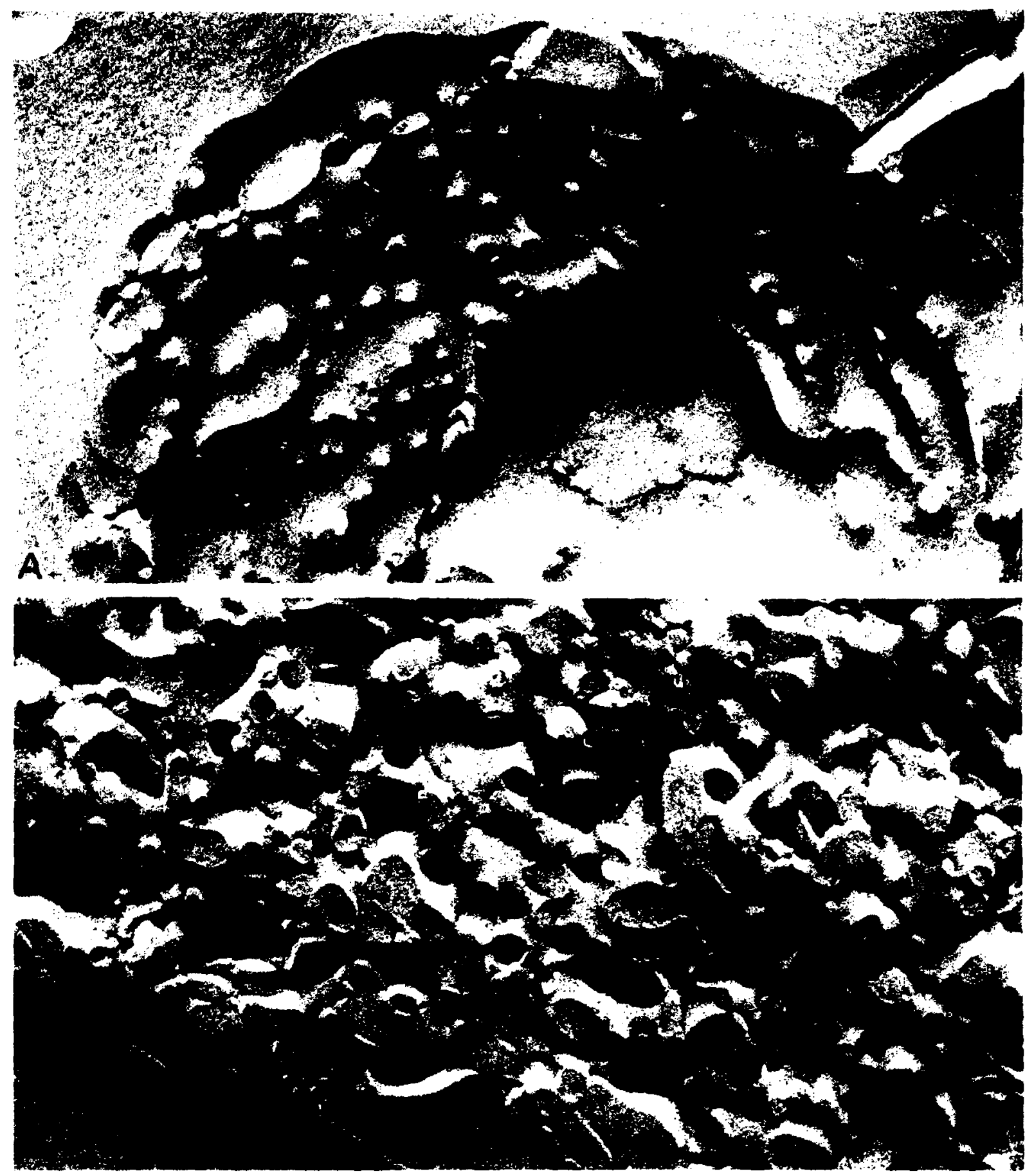

Fig. 7. A and B. Freeze-fracure electron micrographs of an aqueous dispersion of a mixture of gramicidin: $16: 1 / 16: l_{\mathrm{c}}$-phosphatidylcholine $=1: 10$. The sample was quenched conventionally from $25^{\circ} \mathrm{C}$. Magnification approx. $100000 \times$. 
phase when incorporated in $18: 1_{\mathfrak{c}}, 18: 1_{\mathfrak{c}}$-phosphatidylcholine compared to incorporation in 18: $1_{\mathrm{tr}}, 18: 1_{\mathrm{tr}}$-phosphatidylcholine. Dispersions of gramicidin with $16: 1_{c}, 16: 1_{c}$-phosphatidylcholine (1:10) do not show any indication of hexagonally organized lipid. Instead, the ${ }^{31}$ P-NMR spectrum shows a component at $0 \mathrm{ppm}$, which increases at higher temperatures and is not reversible on cooling down, indicating isotropic motion of lipid molecules, next to the majority of the signal evidencing a lamellar lipid organization. Freeze-fracturing of this sample (Fig. 7A,B) shows lipidic particle-containing bilayers next to vast networks of interwoven lipid bilayer and ice fracture planes. The isotropic peak in the mixture with $18: 1_{t r}, 18: 1_{\text {tr }}$-phosphatidylcholine most probably originates from small vesicles, despite the cautious vortexing, since freeze-fracturing showed their presence and no other small lipid structures could be detected.

The chain length-dependent formation of the hexagonal $\mathrm{H}_{I I}$ phase by gramicidin is not re- stricted to unsaturated diacylphosphatidylcholines. ${ }^{31}$ P-NMR spectra of dispersions of gramicidin with various liquid crystalline saturated diacylphosphatidylcholines in a 1:10 molar ratio are presented in Fig. 8. Again, the onset of the induction of the hexagonal $H_{11}$ phase by gramicidin coincides with a fatty acid chain length of 18 carbon atoms. For the shorter chain lengths a complete lamellar phospholipid organization is found. The minor isotropic peaks originate from the occurrence of small bilayer vesicles as evidenced by freeze-fracturing.

The clear fatty acid chain length-dependent nature of the interaction between gramicidin and diacylphosphatidylcholines makes one curious whether similar tendencies would hold in the reversed situation, i.e., varying the length of the peptide helix with a constant fatty acid chain length. From $X$-ray crystallographic studies $[30,31]$ it has been shown that the length of the gramicidin dimer changes from $32 \AA$ to $26 \AA$ upon occupation of the two cation-binding sites in the channel by $\mathrm{Cs}^{+}$or $\mathrm{K}^{+}$. To test whether such a mechanism
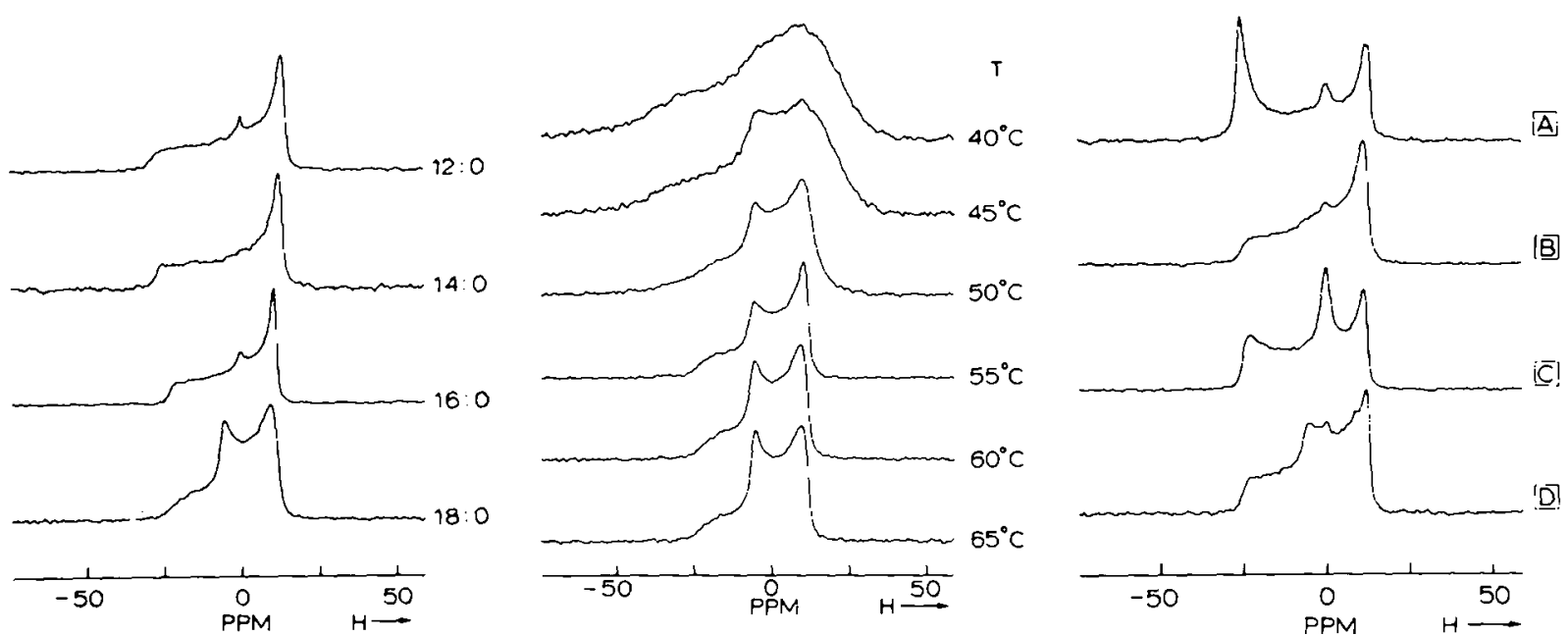

Fig. 8. (Left.) Proton noise decoupled $81.0 \mathrm{MHz}$ "'P-NMR spectra of aqueous dispersions of mixtures of gramicidin with various saturated liquid-crystalline diacylphosphatidylcholines in a 1:10 molar ratio. Fatty acid composition as indicated in the figure. Recording temperatures: $12: 0$ at $30^{\circ} \mathrm{C}, 14: 0$ at $40^{\circ} \mathrm{C}, 16: 0$ at $50^{\circ} \mathrm{C}$ and $18: 0$ at $60^{\circ} \mathrm{C}$.

Fig. 9. (Centre.) Proton noise decoupled $81.0 \mathrm{MHz}$. ${ }^{31} \mathrm{P}-\mathrm{NMR}$ spectra of an aqueous dispersion of a mixture of gramicidin: 18:0/18:0-phosphatidylcholine $=1: 10$ at various temperatures indicated in the figure.

Fig. 10. (Right.) Proton noise decoupled $81.0 \mathrm{MHz}{ }^{31}$ P.NMR spectra of aqueous dispersions of mixtures of: (A) gramicidin: soya phosphatidylcholine $=1: 10$, recorded directly after 3 hours hydrating at $25^{\circ} \mathrm{C} ;(\mathrm{B})$ the same sample as in (A) recorded subsequently after vortexing at $25^{\circ} \mathrm{C}$; (C) the same sample as in (B) recorded subsequently after being heated to $60^{\circ} \mathrm{C}$ and cooled down to $25^{\circ} \mathrm{C}$; (D) gramicidin : egg phosphatidylcholine $=1: 10$, recorded directly after 3 h hydrating at $25^{\circ} \mathrm{C}$. 
could also apply to the lipid-bound gramicidin we have prepared dispersions of gramicidin with $16: 1_{c}, 16: 1_{c}$-phosphatidylcholine, $18: 1_{c}, 18: 1_{c^{-}}$ phosphatidylcholine and $22: 1_{c}, 22: 1_{c}$-phosphatidylcholine with $1: 10$ molar ratios. $\mathrm{K}^{+}$was subsequently added to a final amount, corresponding to an approx. 20-fold excess with respect to the number of cation binding sites of the gramicidin present in the samples. However, in none of these samples did the spectral features change upon addition of $\mathrm{K}^{+}$, suggesting a constant length of the lipid-bound gramicidin dimer, as was indicated also by circular dichroism studies [32].

A change in fatty acid chain length occurs when going from a liquid crystalline to a gel state [33], together with an increase in chain order [34]. To investigate the effect of this transition on the gramicidin-induced hexagonal $\mathrm{H}_{\mathrm{II}}$ phase, 18 : 0,18:0-phosphatidylcholine was a feasible candidate, because of the already demonstrated occurrence of the hexogonal $H_{n}$ phase in the liquid crystalline state of this lipid (see Fig. 8) and its suitable transition temperature of $54^{\circ} \mathrm{C}[35] .{ }^{31} \mathrm{P}$ NMR spectra of dispersions of gramicidin and 18:0,18:0-phosphatidylcholine in a $1: 10$ molar ratio were recorded at various temperatures. The results are shown in Fig. 9. The broad asymmetric spectrum at $40^{\circ} \mathrm{C}$ is typical for gel-state phospholipids organized in extended bilayers $[26,36]$, although theoretically such a spectrum could also arise from hexagonally organized lipid, provided that the correlation time for the diffusion motion around the cylinder axis is long compared to $\Delta \sigma$. However, this latter possibility is highly unlikely in view of the geometry of the lipid molecules in the gel state and the fact that until now hexagonally organized lipid has only been found in the liquid crystalline state. The broadening at the edges of the spectrum is caused by some residual dipolar coupling. The transition to the liquid crystalline state which can be recognized from the diminishing of the dipolar broadening, can be seen to occur around $50^{\circ} \mathrm{C}$ and to be completed around $55^{\circ} \mathrm{C}$. The increase of the gel-to-liquid-crystalline transition width, which for the pure lipid amounts to approx. $1^{\circ} \mathrm{C}[35]$ is in agreement with calorimetric data on gramicidin-16:0,16:0-phosphatidylcholine mixtures [10].

Superimposed on this transition there is a sec- ond transition manifest in which phospholipid molecules enter the hexagonal $\mathrm{H}_{11}$ phase. From $60^{\circ} \mathrm{C}$ the amount of hexagonally organized lipid does not increase appreciably. But already in a gel state environment at temperatures as low as 40 and $45^{\circ} \mathrm{C}$ phospholipid molecules are organized in a hexagonal $\mathrm{H}_{11}$ phase. Hexagonally organized lipid in a gel state environment was also found in mixtures of gramicidin and $18: 1_{\mathrm{tr}}, 18: 1_{\mathrm{tr}}$ - - hosphatidylethanolamine [17].

As the length represented by the 18 carbon atoms-long fatty acids employed in this study, at which the onset of the induction of the hexagonal $\mathrm{H}_{11}$ phase by gramicidin occurs, is most common in biological membranes we have also tested some phosphatidylcholines from natural sources, e.g., soya beans and hen eggs. The predominant fatty acid species in soya phosphatidylcholine are linoleic $(18: 2 \mathrm{c})$ and palmitic $(16: 0)$ acid; those in egg phosphatidylcholine are oleic $\left(18: l_{c}\right)$ and palmitic $(16: 0)$ acid. In Fig. 10 are shown ${ }^{31}$ P-NMR spectra of dispersions of mixtures of gramicidin with soya phosphatidylcholine or egg phosphatidylcholine in a 1:10 molar ratio. The spectra of the soya phosphatidylcholine-gramicidin mixture after hydration and gentle vortexing (Fig. 10A) showed some unexpected features. Next to the high-field peak at approx. $12 \mathrm{ppm}$, feature of the asymmetric spectrum indicating lamellar lipid organization, and an isotropic peak at $0 \mathrm{ppm}$, there is a strongly enhanced intensity at the low-field shoulder position at approx. $-26 \mathrm{ppm}$. The shoulder resonance position corresponds with the time-averaged tensor component $\sigma_{1}$, which originates from lamellar organized phospholipid molecules oriented parallel to the magnetic field. Therefore, the enhanced intensity at $-26 \mathrm{ppm}$ most probably indicates a preferential orientation of part of the soya phosphatidylcholine molecules. After vigorously vortexing for $3 \mathrm{~min}$, this preferential orientation is disrupted and a 'normal' ${ }^{31} \mathrm{P}-\mathrm{NMR}$ spectrum results (Fig. 10B), that indicates a lamellar organization with small components indicating very little amounts of phospholipid molecules in hexagonal and isotropic phases. When this sample is heated to $60^{\circ} \mathrm{C}$ for $5 \mathrm{~min}$ and subsequently cooled down again to $25^{\circ} \mathrm{C}$ the preferential orientation is partly rstored. The freeze-fracture morphology of this sample is similar to that of the $16: 1_{c}, 16: 1_{c}$-phos- 
phatidylcholine-gramicidin mixtures shown in fig. 7. A sample of an egg phosphatidylcholinegramicidin mixture showed the anomalous spectral features also, but less pronounced and only at temperatures over $50^{\circ} \mathrm{C}$. At $25^{\circ} \mathrm{C}$ a spectrum resulted which is shown in Fig. 10D.

The preferential orientation encountered here, which occasionally and to a minor extent also has been found with $18: 1_{c}, 18: 1_{c}$-phosphatidylcholine-gramicidin mixtures (see Fig. 6), is difficult to explain. It is apparently mechanically unstable and depends on the presence of gramicidin, the lipid environment and temperature. Also the strength and/or the direction of the magnetic field appears to be involved, since these phenomena were not found with a Bruker WH 90 NMR spectrometer, operating at $36.4 \mathrm{MHz}$, in which the direction of the magnetic field is perpendicular to the long axis of the NMR tube, as opposed to the parallel direction of the WP 200 field. Whether the axis of the gramicidin helix orients in the magnetic field or other defined structural features are involved must await further experiments.

However, despite the anomalous NMR behaviour, the freeze-fracture and NMR results clearly indicate that gramicidin can also induce hexagonal and other nonbilayer structures in natural lipids.

\section{Discussion}

The main observation made in this study is that gramicidin is able to induce a hexagonal $\mathrm{H}_{\mathrm{II}}$-phase organization in diacylphosphatidylcholines and that it does so in a fatty acid chain length-dependent way.

The onset of the fatty acid chain length-dependent formation of the hexagonal $\mathrm{H}_{11}$ phase by gramicidin has been shown to coincide with a length exceeding 16 carbon atoms long fatty acids. From neutron diffraction studies [33] the bilayer thickness of liquid crystalline 16:0,16:0-phosphatidylcholine may be estimated to be $42-43 \AA$. Assuming the thickness of the polar part to be about the same as in the gel state, approx. $6 \AA$, a thickness of the hydrophobic part results of 30-31 $\AA$. When incorporated in a lipid environment in peptide:lipid molar ratios under $1: 5$, gramicidin adopts a dimeric conformation which is different from the one in organic solvents [32]. The length of the gramicidin channel in a lipid environment in the presence of monovalent cations seems larger than in the corresponding crystal structure ( $26 \AA \dot{A})$, but shorter than the crystal structure without monovalent cations ( $32 \AA$, Refs. $30,31,37)$. The apparently ion-dependent length of lipid associated gramicidin [32] of approx. $30 \AA$ concurs remarkably with the hydrophobic thickness mentioned above.

When the length of the hydrophobic part of the phospholipid molecules exceeds this length, apparently an unstable situation arises which results in the formation of a hexagonal $\mathrm{H}_{11}$ phase for part of the molecules. The longer the fatty acid chains, the more lipid molecules enter the hexagonal $\mathrm{H}_{\text {II }}$ phase, probably because with longer fatty acids, less lipid molecules are necessary to accommodate the gramicidin in the hexagonal phase. From the concentration-dependence of the gramicidin-induced hexagonal phase a gramicidin enrichment in this phase may be inferred. although no clear stoicheiometry for a hexagonal complex can be deduced. From the approximately linear parts in Fig. 5 at low gramicidin concentrations, a rough estimate yields a peptide:lipid ratio of $1: 7$ till $1: 10$ in the hexagonal phase, but this probably does not hold at higher gramicidin contents.

Although the fatty acid chains in gel-state lipids are longer than in the liquid-crystalline state [33], gramicidin does not induce a hexagonal organization in the gel state, most likely because rigid fatty acid chains do not fit in a hexagonal phase. On the other hand, the fluidizing properties of gramicidin [10] are clearly apparent from the presence of a hexagonal phase in a gel-state environment (see Fig. 9).

That next to the length of the fatty acids, on a second level also the volume or shape of the hydrophobic part of the phosphatidylcholine plays a role in the extent of the induction of the hexagonal phase by gramicidin, can also be seen when for instance in Fig. 6, 18: $1_{\mathrm{tr}}, 18: 1_{\mathrm{tr}}$-phosphatidylcholine is compared with $18: 1_{c}, 18: 1_{c}$-phosphatidylcholine. The higher amount of hexagonal phase in the latter is evident. Nevertheless, the length of the $18: 1_{\text {tr }}$-fatty acid may be estimated to be equal or even slightly longer than the length of the 18: $1_{\mathrm{c}}$-fatty acid. However, monolayer studies show 
that the area of the $18: 1_{\text {tr }}, 18: 1_{\text {tr }}$-phosphatidylcholine molecule is smaller than the area of the 18: $1_{c}, 18: 1_{c}$-phosphatidylcholine $\left(61 \AA^{2} / \mathrm{mol}\right.$ vs. $68 \AA^{2} / \mathrm{mol}$ at $23^{\circ} \mathrm{C}$ and $30 \mathrm{mN} / \mathrm{m}$; R.A. Demel, personal communication). This makes the $18: I_{\text {tr }}$ lipid more cylindrical than the $18: 1_{\mathrm{c}}$-analog and therefore possibly less suitable to fit in a hexagonal phase.

The transition from a lamellar to a hexagonal $\mathrm{H}_{11}$ phase of unsaturated phosphatidylethanolamines has been shown to be clearly temperaturedependent [38], most probably as a result of a growing imbalance between hydrophobic and hydrophilic areas. The amount of hexagonal $\mathrm{H}_{n}$ phase and the corresponding distribution of gramicidin over hexagonal and lamellar phases in phosphatidylcholines is not temperature-dependent and solely determined by the type of fatty acid and the gramicidin concentration. However, increasing temperature would also here be expected to increase hydrophobic areas and therefore increase the amount of hexagonal $\mathrm{H}_{\mathrm{II}}$ phase. That we do not observe such an increase may be related to the fatty acid chain ordering influence of gramicidin as found for liquid crystalline $14: 0,14: 0$ - and 16:0,16:0-phosphatidylcholine [11,12].

The lamellar lipid organization found in previous studies on gramicidin with 14:0,14:0- and $16: 0,16: 0$-phosphatidylcholine $[10,18]$ is in agreement with our data on these systems. The isotropic ${ }^{31} \mathrm{P}-\mathrm{NMR}$ signals found at extremely high gramicidin contents [18], where gramicidin possibly adopts a different conformation [32] as a consequence of peptide-peptide interaction, are reflected in the more symmetrical line-shapes we have found at these high gramicidin concentrations.

That the structure of the polypeptide itself also plays a crucial role in the bilayer destabilization is indicated by the observations [9] that the intrinsic membrane protein glycophorin stabilizes bilayer structure in various model membrane systems.

To accomodate the gramicidin molecule in the hexagonal $\mathrm{H}_{\text {II }}$ phase, one may think of several possibilities. The observed effects could relate to gramicidin either in a dimeric or a monomeric conformation. Differences in circular dichroism spectra of gramicidin incorporated in 18:0,18:0phosphatidylcholine but also in 16:0,16:0-phos- phatidylcholine compared to shorter chain lengths have been interpreted as a decrease of the dimerization constant [32]. Unfortunately these studies have only been done in the lipid gel state, where the molecules do not have the possibility to orient themselves differently, e.g., in a hexagonal phase (see Fig.9). On the other hand, the gramicidin monomer possesses a conical shape due to the large tryptophan residues, all on one side of the molecule, near the channel entrance. This shape would fit nicely in a hexagonal $\mathrm{H}_{11}$ phase, with the long axis of the molecule directed towards the aqueous cylinder, provided that the orientation of the gramicidin molecule is reversed, the tryptophan residues now near the centre of the hydrophobic domain. To gain more insight in these possible orientations studies with desformylgramicidin and the covalently bonded dimer are in progress.

Although during a transition from a lamellar to a hexagonal structure the gramicidin dimer probably breaks up, we favour as yet for the hexagonal phase itself the model depicted in Fig. 11, where gramicidin dimers span adjacent cylinders. This

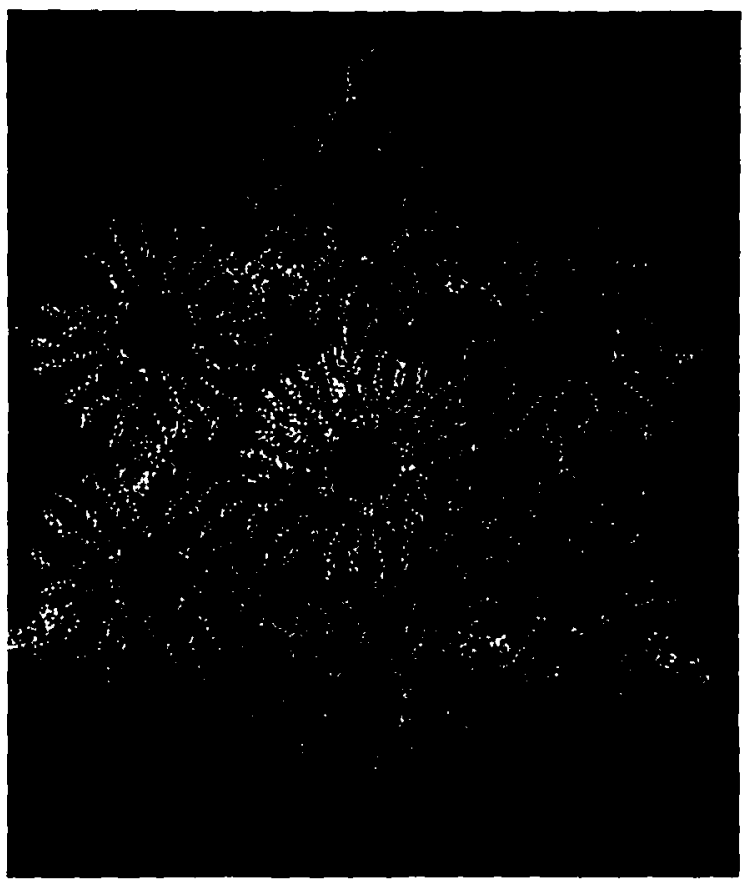

Fig. 11. Tentative model of the gramicidin-22: $l_{c} / 22: l_{c}$-phosphatidylcholine complex in the hexagonal $H_{11}$ phase. 
model resembles most the bilayer orientation of gramicidin, allows for intermolecular hydrogen bonding and is supported by the comparison of the length of the dimer and the thickness of the hydrophobic parts. Fig. 11 represents a cross-section through a collection of hexagonal $\mathrm{H}_{\text {II }}$ cylinders of $22: 1_{c}, 22: 1_{c}$-phosphatidylcholine with gramicidin in a 9:1 molar ratio. For the $\pi_{6}(L, D)$ conformation of gramicidin this ratio corresponds approximately with a single layer of lipid molecules around each gramicidin molecule [10] and is in agreement with the estimated stoicheiometry from Fig. 5. Due to the applied regularity and the two-dimensional character of the model, the central cylinder does not contain gramicidin. In a threedimensional model it is most likely that the gramicidin dimers are more evenly distributed in the hexagonal cylinders like the steps of winding stairs. Another feature revealed by the model is that arranging 14 phosphatidylcholine molecules in the central cylinder, which is easily possible with the corresponding phosphatidylethanolamines, can only be accomplished under highly restrictive conditions. On the other hand, it can be seen that the presence of gramicidin relieves this constraint and furnishes the conditions to arrange the choline moieties easily around the aqueous channels.

As a third possibility, one may think of an orientation of the gramicidin monomers or dimers in the hydrophobic interior of the hexagonal phase with the long axis of the gramicidin molecule parallel to the hexagonal-phase cylinders. However, this explains less well the good correlation between the length of the gramicidin dimer and the length of the lipid molecules at which the onset of the hexagonal $\mathrm{H}_{11}$ phase formation occurs. Furthermore, $\Delta \sigma$ of the gramicidin-phosphatidylcholine hexagonal phase is reduced, compared to $\Delta \sigma$ of the hexagonal phase of pure phosphatidylethanolamine. Most likely this is caused by additional modes of motion, although conformational changes cannot be excluded. Anyhow, it indicates that the polar head groups are sensing the presence of gramicidin, which is unlikely when the gramicidin molecules are buried parallel in the hydrophobic interior.

Extended areas of hexagonal $\mathrm{H}_{1 \mathrm{I}}$-phase cylinders are difficult to reconcile with the barrier function of membranes, although single cylinders or a locally induced transitory hexagonal phase could well serve several functional purposes of those membranes [39]. But it is interesting to see that with phosphatidylcholines possessing intermediate lengths and/or hydrophobic volumes like soya phosphatidylcholine and 16:1 $1_{c}, 16: 1_{c}$-phosphatidylcholine, upon incorporation of gramicidin, intermediate structures are formed characterized by isotropic ${ }^{31} \mathrm{P}-\mathrm{NMR}$ signals and visualized by freeze-fracturing as bilayer associated lipid particles and interwoven networks of ice and lipid bilayer fracture planes. Especially so, when realising that these intermediate lengths are most common in biological membranes. The occurrence of lipidic particles has been reported before in mixtures containing hexagonal $\mathrm{H}_{11}$-phase-preferring lipids [40-43] as intermediates in the transition from a lamellar to a hexagonal organization $[44,45]$, and to be associated with vesicle fusion [44,46-48]. In recent work on the $\mathrm{Ca}^{2+}$-dependent polymorphism of cardiolipin (unpublished data) both the occurrence of lipidic particles as a result of fusion events and the interwoven network of ice and lipid fracture planes were found as intermediaries between the lamellar and hexagonal phase. Based on visual appearance, ${ }^{31} \mathrm{P}-\mathrm{NMR}$ and small-angle $\mathrm{X}$-ray diffraction the latter intermediary has been interpreted as a cubic phase which, according to the freeze-fracturing data, which were similar to those found in this study, could relate to 'Schwarz's primitive cubic minimal surface' structure (see for instance Ref. 49). Cubic phases [50] and lipidic particles [42,43] have also been reported for some glycolipid-containing model membrane systems. Although little is known about the functional significance of the cubic phases, it is intriguing to note that some biological membrane systems, such as the prolamellar body of developing chloroplasts [51], have a morphology which greatly resembles that of a cubic phase [49]. The observation that gramicidin does induce similar structures in soya phosphatidylcholine and $16: 1_{c}, 16: 1_{c}$-phosphatidylcholine dispersions suggests that peptide-lipid interaction may contribute to the formation of such structures.

In summary, the present study clearly demonstrates that peptide-lipid interactions can largely influence the phase structure of membrane lipids. 
In particular, the observation that a hydrophobic peptide can induce the formation of various nonbilayer lipid structures opens exciting new perspectives in various areas of membrane biology such as fusion and anchoring and insertion of membrane proteins. With respect to this latter possibility, it is intriguing to note that gramicidin. when added to membrane systems, spontaneously inserts itself as a transmembrane dimer, as can be inferred from the observed permeability changes. Furthermore, the idea that a mismatch in length of the hydrophobic domain of peptide and membrane may trigger phase changes, which could be important for the insertion of a peptide, bears direct relation to some models $[52,53]$ proposed for the insertion of the newly formed peptide chain of some membrane or secretory proteins.

Whether non-bilayer lipid structures are involved in these processes is presently under active investigation.

\section{Acknowledgements}

We would like to thank Dr. $H$. Rijnbout for performing the X-ray experiments and Dr. T.F. Taraschi for correcting the English. The lipids were skilfully synthesized, isolated and purified by Mr. W.S.M. Geurts van Kessel and Ms. M. Tieman. The expert assistance of Dr. J.L.M. Leunissen in molecular model photography is gratefully acknowledged.

\section{References}

1 Cullis, P.R. and De Kruijf, B. (1979) Biochim. Biophys. Acta $551,399-420$

2 De Kruijff, B., Cullis, P.R. and Verkleij, A.J. (1980) Trends Biochem. Sci. 5, 79-83

3 Burnell, E.E., Van Alphen, L., Verkleij, A.J. and De Kruijff, B. (1980) Biochim. Biophys. Acta 597, 492-501

4 Stier, A., Finch, S.A.E. and Bösterling, B. (1978) FEBS Lett. 91, 109-112

5 De Kruijff, B., Van den Besselaar, A.M.H.P., Cullis, P.R., $V$ an den Bosch, H. and Van Deenen, L.L.M. (1978) Biochim. Biophys. Acta 514, 1-8

6 De Grip. W.J., Drenthe, E.H.S., Van Echteld, C.J.A., De Kruijff, B. and Verkleij, A.J. (1979) Biochim. Biophys. Acta 558. 330-337

7 De Kruijff, B. and Cullis, P.R. (1980) Biochim. Biophys. Acta $602,477-490$

8 De Kruijff, B. and Cullis, P.R. (1980) Biochim. Biophys. Acta 601, 235-240
9 Taraschi, T., De Kruijff, B., Verkleij, A.J. and Van Echteld, C.J.A. (1982) Biochim. Biophys. Acta 685, 153-161

10 Chapman, D., Cornell, B.A., Eliasz, A.W. and Perry, A. (1977) J. Mol. Biol. 113, 517-538

11 Cortijo, M. and Chapman, D. (1981) FEBS Lett. 131. 245-248

12 Rice. D. and Oldfield, E. (1979) Biochemistry 18. 3272-3279

13 Urry, D.W. (1971) Proc. Natl. Acad. Sci. U.S.A. 68, 672-676

14 Urry, D.W., Goodall, M.C., Glickson, J.D. and Mayers. D.F. (1971) Proc. Natl. Acad. Sci. U.S.A. 68, 1907-1911

15 Weinstein, S., Wallace, B.A., Blout. E.R., Morrow, J.S. and Veatch, W. (1979) Proc. Natl. Acad. Sci. U.S.A. 76, 42304234

16 Weinstein. S., Wallace, B.A.. Morrow, J.S. and Veatch, W.R. (1980) J. Mol. Biol. 143, 1-19

17 Van Echteld, C.J.A., Van Stigi, R., De Kruijf,, B., Leunissen-Bijvelt, J., Verkleij. A.J. and De Gier, J. (1981) Biochim. Biophys. Acta 648, 287-291

18 Rajan, S., Kang, S.-Y., Gutowsky, H.S. and Oldfield, E. (1981) J. Biol. Chem. 256, 1160-1166

19 Van Deenen, L.L.M. and De Haas, G.H. (1964) Adv. Lipid Res. 2, 168-363

20 Geurts van Kessel, W.S.M., Tieman, M. and Demel, R.A. (1981) Lipids 16, 58-63

21 Ververgaert, P.H.J.Th., Verkleij, A.J., Elbers, P.F. and Van Deenen, L.L.M. (1973) Biochim. Biophys. Acta 311. 320324

22 Müller, M., Meister, N. and Moor, H. (1980) Mikroskopie 36. $129-140$

23 Van Venetiè, R., Hage, W.J., Bluemink, J.G. and Verkleij, A.J. (1981) J. Microsc. 123, 287-292

24 Seelig, J. (1978) Biochim. Biophys. Acta 515, 105-140

25 Brown, M.F. and Seelig, J. (1978) Biochemistry 17, 381-384

26 Cullis, P.R., De Kruijff, B. and Richards, R.E. (1976) Biochim. Biophys. Acta 426, 433-446

27 Cullis, P.R. and De Kruijf, B. (1978) Biochim. Biophys. Acta 507, 207-218

28 Cullis, P.R., Verkleij, A.J. and Ververgaert, P.H.J.Th. (1978) Biochim. Biophys. Acta 513, 11-20

29 Burnell, E.E., Cullis, P.R. and De Kruijff, B. (1980) Biochim. Biophys. Acta 603, 63-69

30 Koeppe, R.E., Hodgson, K.O. and Stryer, L. (1978) J. Mol. Biol. 121, 41-54

31 Koeppe, R.E., Berg, J.M.. Hodgson, K.O. and Stryer, L. (1979) Nature 279, 723-725

32 Wallace, B.A., Veatch, W.R. and Blout, E.R. (1981) Biochemistry $20,5754-5760$

33 Büldt, G., Gally, H.U., Seelig, A.. Seelig, J. and Zaccai, G. (1978) Nature 271, 182-184

34 Gaber, B.G. and Peticolas, W.L. (1977) Biochim. Biophys. Acta 465, 260-274

35 Hinz, H.J. and Sturtevant, J.M. (1972) J. Biol. Chem. 247. 6071-6075

36 Seelig, J. and Gally, H.U. (1976) Biochemistry 15, 51995204

37 Lrry, D.W., Prasad, K.U. and Trapane, T.L. (1982) Proc. Natl. Acad. Sci. U.S.A. 79, 390-394

38 Cullis, P.R. and De Kruijff, B. (1978) Biochim. Biophys. Acta 513, 31-42 
39 Kachar, B. and Reese, T.S. (1982) Nature 296, 464-466

40 Verkleij, A.J., Mombers, C., Leunissen-Bijvelt, J. and Ververgaert, P.H.J.Th. (1979) Nature 279, 162-163

41 De Kruijff, B., Verkleij, A.J., Van Echteld, C.J.A., Gerritsen, W.J., Mombers, C.. Noordam, P.C. and De Gier, J. (1979) Biochim. Biophys. Acta 555, 200-209

42 Sen, A., Williams, W.P., Brain, A.P.R., Dickens, M.J. and Quinn, P.J. (1981) Nature 293, 488-490

43 Sen, A., Williams, W.P., Brain, A.P.R. and Quinn. P.J. (1982) Biochim. Biophys. Acta 685, 297-306

44 Vail, W.J. and Stollery, J.G. (1979) Biochim. Biophys. Acta 551, 74-84

45 Van Venetië, R. and Verkleij, A.J. (1981) Biochim. Biophys. Acta 645, 262-269

46 Verkleij, A.J., Mombers, C., Gerritsen, W.J., Leunissen-Bi- jvelt, J. and Cullis, P.R. (1979) Biochim. Biophys. Acta 555. 358-361

47 Verkleij, A.J., Van Echteld, C.J.A., Gerritsen. W.J., Cullis, P.R. and De Kruijff, B. (1980) Biochim. Biophys. Acta 600 . 620-624

48 Verkleij, A.J., De Maagd. R., Leunissen-Bijvelt, J. and De Kruijf, B. (1982) Biochim. Biophys. Acta 684, 255-262

49 Larsson, K., Fontell, K. and Krog. N. (1980) Chem. Phys. Lipids 27, 32!-328

s0 Wieslander, A., Rilfors, L., Johansson, L.B. and Lindblom, G. (1981) Biochemistry 20, 730-735

51 Simpson, D.J. (1978) Carlsberg Res. Commun. 43, 145-170

52 Wickner, W. (1979) Annu. Rev. Biochem. 48, 23-45

53 Engelman, D.M. and Steitz. T.A. (1981) Cell 23, 411-422 